6. Демьянов В. Ф., Малоземов В. Н. Введение в минимакс. Москва : Наука, $1972.368 \mathrm{c}$.

\title{
SOLUTION OF THE PROBLEM OF RESTORING DISCONTINUOUS FUNCTIONS BY THE MINIMAX
}

The article suggests a method for approximating a function of one and two variables with discontinuities of the first kind by a discontinuous approximation spline. The experimental data are the one-sided boundaries of the given nodes. To solve this problem in this paper, we use the minimax method.

Key words: discontinuous functions, discontinuous spline, approximation, interpolation, minimax.

Одержано 14.02.2019

\section{УДК 519.1}

DOI: $10.32626 / 2308-5878.2019-19.104-111$

В. І. Петренюк, канд. фіз.-мат. наук, доцент

Центральноукраїнський національний технічний університет, м. Кропивницький

\section{СТРУКТУРА 20-ТИ 9-ТИ ВЕРШИННИХ ГРАФІВ-ОБСТРУКЦІї ТОРА}

\author{
Досліджено структуру решти 9-ти вершинних графів- \\ обструкцій для тору.
}

Ключові слова: граф-обструкиія, тор, ф-перетворення графів.

Вступ. Основні визначення та позначення взято з [1]. У роботі [2] запропоновано спосіб побудови графів-обструкцій обмеженого орієнтованого роду як $\varphi$-образу двох графів, один з яких має бути квазізіркою, з'єднаних шляхом ототожнення пар вершин, для випадку несуттєвості порядку ототожнення зазначених пар точок; тобто один із підграфів породжених підмножинами точок допускатиме перестановку довільної пари тачок з'єднання, наприклад, є повним. Цей підхід може видавати такі графи, які набуватимуть статус обструкцій після стискання в точку усіх лишніх ребер-променів квазізірки, саме так побудовані зазначені графи. Однак не всі графи-обструкції для тору можливо отримати цим способом. Однією з причин відсутності лишніх ребер $є$ наявність двостороннього доступу до деяких точок із тих пар точок, що підлягають ототожненню в точку-вершину графа.

Задача полягатиме у завершенні розпочатої в [4] роботи по вивченню структури 9-ти вершинних графів-обструкцій для тору, наве- 
дених у [3] для використання при побудові n-вершинних, $n>9$, графів-обструкцій для тору.

Лема 1. Виконуються наступні твердження:

1) $D_{32} \in \varphi$-образом графів $K_{6} \backslash K_{2}^{1}$ та квазізірки $H \quad 3$ центром $K_{2,3}$, де $K_{6}^{0} \backslash K_{2}^{1}=\left\{i^{\prime}\right\}_{1}^{6}, K_{6}^{1} \backslash K_{2}^{1}=K_{6}^{1} \backslash\left\{\left(1^{\prime}, 3^{\prime}\right),\left(2^{\prime}, 5^{\prime}\right)\right\}, H^{0}=\left\{i^{\prime \prime}\right\}_{1}^{6} \cup\{a, b, v\}$, $H^{1}=K_{2,3}^{1} \cup\left\{(2 ", a),(b, 3 "),\left(b, 1^{\prime \prime}\right),(b, 5 ")\right\}$, при перетворенні заданому формулою: $\varphi\left(K_{6} \backslash K_{2}^{1}+H, \sum_{i=1}^{6}\left(i^{\prime}+i^{\prime \prime}\right)\right) \rightarrow\left(D_{32},\{i\}_{i=1}^{6}\right)$ та виконаному шляхом ототожнення усіх пар $\left(i^{\prime}, i^{\prime \prime}\right)$ вершин з множин $M^{\prime}=\left\{i^{\prime}\right\}_{1}^{6}$ та $M^{\prime \prime}=\{i "\}_{1}^{6}$;

2) $D_{33}$ - граф-обструкція для тору $€ \varphi$-образом графів $K_{5}$ та $H \cup S t_{3}(b), \quad$ де $H$-квазізірка 3 центром $K_{2,3}, \quad K_{5}^{0}=\left\{i^{\prime}\right\}_{1}^{5}$, $H^{1}=K_{2,3}^{1} \cup\left\{\left(1^{\prime \prime}, a\right),\left(v, 3^{\prime \prime}\right),\left(c, 5^{\prime \prime}\right)\right\}, \quad H^{0}=\left\{i{ }^{\prime \prime}\right\}_{1}^{5} \cup\{a, c, v\}, \quad S t_{3}(b)-$ проста зірка з центром $\mathrm{b}, S t_{3}^{0}(b)=\{i "\}_{1}^{3}$, при перетворенні заданому наступною формулою: $\varphi\left(K_{5}+H \cup S t_{3}(b), \sum_{i=1}^{5}\left(i^{\prime}+i^{\prime \prime}\right)\right) \rightarrow\left(D_{33},\{i\}_{i=1}^{5}\right)$ та виконаному шляхом ототожнення усіх пар $\left(i^{\prime}, i\right.$ ) вершин 3 множин $M^{\prime}=\left\{i^{\prime}\right\}_{1}^{5}$ та $M^{\prime \prime}=\{i "\}_{1}^{5}$;

3) $D_{34} \in \quad \varphi$-образом графів $K_{6} \backslash K_{3}^{1} \quad$ та $H$, де $K_{6}^{0}=\left\{i^{\prime}\right\}_{1}^{6}$, $K_{6}\left(\left\{i^{\prime \prime}\right\}_{i=4}^{6}\right)=\overline{K_{3}}, H^{0}=\left\{i^{\prime \prime}\right\}_{1}^{6} \cup\{a, b, c\}, K_{6}\left(\left\{i^{\prime \prime}\right\}_{i=}^{3}\right)=K_{3}, H^{1}=K_{5}^{1} \backslash$ $\backslash\{(c, 1 "),(c, 4 ")\} \cup\left\{(c, 6 "),\left(1^{\prime \prime}, 6^{\prime \prime}\right),(d, 1 "),\left(2^{\prime \prime}, b\right),(3 ", a),\left(5^{\prime \prime}, c\right)\right\}$, де вершина 6" розділяє ребро $(c, 1 ")$ графа $K_{5} \backslash(c, 4 ")$, при перетворенні заданому: $\varphi\left(K_{6} \backslash K_{3}^{1}+H, \sum_{i=1}^{6}\left(i^{\prime}+i "\right)\right) \rightarrow\left(D_{34},\{i\}_{i=1}^{6}\right)$ шляхом ототожнення усіх пар $\left(i^{\prime}, i^{\prime \prime}\right)$ з множин приєднання $M^{\prime}=\left\{i^{\prime}\right\}_{1}^{6}, M^{\prime \prime}=\left\{i{ }^{\prime \prime}\right\}_{1}^{6}$;

4) $D_{35} \quad \varphi$-образ графів $K_{6} \backslash\left(K_{3}^{1}+K_{2}^{1}\right) \quad$ та $H$, де $K_{6}^{0}=\left\{i^{\prime}\right\}_{1}^{6}$, $K_{6}\left(\left\{i^{\prime \prime}\right\}_{i=4}^{6}\right)=\overline{K_{3}}, \quad K_{6}\left(\left\{i^{\prime \prime}\right\}_{i=}^{3}\right)=K_{3} \backslash\left(4^{\prime}, 5^{\prime}\right), \quad H^{0}=\left\{i^{\prime \prime}\right\}_{1}^{6} \cup\{a, b, v\}$, $H^{1}=K_{2,3}^{1} \cup\left\{(b, 2 "),\left(4^{\prime \prime}, v\right),\left(3^{\prime \prime}, a\right),\left(5^{\prime \prime}, v\right)\right\}$, при перетворенні заданому: $\varphi\left(K_{6} \backslash\left(K_{3}^{1}+K_{2}^{1}\right)+H, \sum_{i=1}^{6}\left(i^{\prime}+i^{\prime \prime}\right)\right) \rightarrow\left(D_{35},\{i\}_{i=1}^{6}\right) \quad$ ототожненням усіх пар $\left(i^{\prime}, i^{\prime \prime}\right)$ з множин приєднання $M^{\prime}=\left\{i^{\prime}\right\}_{1}^{6}, M^{\prime \prime}=\left\{i^{\prime \prime}\right\}_{1}^{6}$. 


\section{Лема 2.}

1) $D_{36}$ - обструкція для тору $є \varphi$-образом графів $K_{6} \backslash K_{3}^{1}$ та $H$, де $K_{6}^{0}=\left\{i^{\prime}\right\}_{1}^{6}, K_{6}\left(\left\{i^{\prime \prime}\right\}_{i=4}^{6}\right)=\overline{K_{3}}, K_{6}\left(\left\{i^{\prime \prime}\right\}_{i=}^{3}\right)=K_{3}, H^{0}=\left\{i^{\prime \prime}\right\}_{1}^{6} \cup\{a, b, v\}$, $H^{1}=K_{4}^{1} \backslash\{(a, v)\} \cup\left\{\left(1^{\prime}, a\right),\left(v, 1^{\prime \prime}\right),\left(4^{\prime \prime}, b\right),\left(5^{\prime \prime}, b\right),(2 ", a),\left(3^{\prime \prime}, v\right)\right\}$, де вершина 1 " розділяє ребро $(a, v)$ графа $K_{4}$, при перетворенні заданому наступною формулою: $\varphi\left(K_{6} \backslash K_{3}^{1}+H, \sum_{i=1}^{6}\left(i^{\prime}+i^{\prime \prime}\right)\right) \rightarrow\left(D_{36},\{i\}_{i=1}^{6}\right)$ шляхом ототожнення усіх пар $\left(i^{\prime}, i^{\prime \prime}\right)$ вершин 3 множин приєднання $M^{\prime}=\left\{i^{\prime}\right\}_{1}^{6}, M^{\prime \prime}=\left\{i^{\prime \prime}\right\}_{1}^{6}$;

2) $D_{37}$ - обструкція для тору є $\varphi$-образом графів $K_{3,3}$ та $H$, де $K_{3,3}^{0}=\left\{i^{\prime}\right\}_{1}^{6}, \quad K_{3,3}\left(\left\{i^{\prime \prime}\right\}_{i=4}^{6}\right)=\overline{K_{3}}, \quad K_{3,3}\left(\left\{i^{\prime \prime}\right\}_{i=1}^{3}\right)=K_{3}, \quad H^{1}=K_{4}^{1} \cup$ $\cup\left\{\left(5^{\prime \prime}, a\right), \cup\left\{\left(5^{\prime}, a\right),(a, 4 "),\left(2^{\prime \prime}, b\right),\left(5^{\prime \prime}, b\right),(2 ", c),(4 ", c),(a, 1 "),(a, 3 ")\right\}\right.$, $H^{0}=\{i "\}_{1}^{6} \cup \cup\{a, b, c\}, H\left\{a, b, c, 6^{\prime \prime}\right\}=K_{4}$, де вершини $5 ", 4 ", 2 "$ розділяють ребра $(a, b),(c, b),(a, c)$ графа $K_{4}$ (із трьома кратними ребрами), при перетворенні заданому: $\varphi\left(K_{3,3}+\right.$ $\left.+H, \sum_{i=1,}^{6}\left(i^{\prime}+i^{\prime \prime}\right)\right) \rightarrow\left(D_{37},\{i\}_{i=1}^{6}\right)$ шляхом ототожнення усіх пар $\left(i^{\prime}, i^{\prime \prime}\right)$ вершин з множин приєднання $M^{\prime}=\left\{i^{\prime}\right\}_{1}^{6}, M^{\prime \prime}=\left\{i^{\prime \prime}\right\}_{1}^{6}$;

3) $D_{38}$ - граф-обструкція для тору $\epsilon$-образом графів $K_{5}$, $K_{5}^{0}=\left\{i^{\prime}\right\}_{1}^{7}$, де вершини $6^{\prime}, 7^{\prime}$ розділяють ребро $\left(4^{\prime}, 5^{\prime}\right)$, та $H$, де $H$ - квазізірка 3 центром $K_{5} \backslash e, H^{0}=\{i "\}_{2}^{5} \cup\{a, 7 ", 6 ", v\}$, $H\left(\left\{a, 5^{\prime \prime}, 6^{\prime \prime}, 7 ", 4 ", v\right\}\right) \cong K_{4}, H^{1}=K_{5}^{1} \backslash\{(v, 6 "),(a, 7 ")\} \cup\{(3 ", a),(v, 4 ")$, $(6 ", 4 "),(a, 5 "),(7 ", 5 ")\}$, при перетворенні заданому: $\varphi\left(K_{5}+\right.$ $\left.+H, \sum_{i=2}^{7}\left(i^{\prime}+i "\right)\right) \rightarrow\left(D_{38},\{i\}_{i=2}^{7}\right)$ та виконаному шляхом ототожнення усіх пар $\left(i^{\prime}, i^{\prime \prime}\right)$ вершин з $M^{\prime}=\left\{i^{\prime}\right\}_{2}^{7}$ та $M^{\prime \prime}=\{i "\}_{2}^{7}$;

4) $D_{39} \in \varphi$-образом графів $K_{5}, K_{5}^{0}=\left\{i^{\prime}\right\}_{1}^{5}$, та $H$, де $H$ - квазізірка із центром $C_{4}$ - простим циклом довжини 4, де $C_{5}^{1}=\{(a, b)$, $(v, b),(a, c),(c, v)\}, H^{1}=C_{5}^{1} \cup\left\{\left(5^{\prime \prime}, a\right),\left(5^{\prime \prime}, b\right),\left(4^{2}, a\right),\left(4^{\prime \prime}, b\right),(v, 1 "),\left(v, 3^{\prime \prime}\right)\right.$, $\left.(c, 1 "),\left(c, 3^{\prime \prime}\right),\left(v, 2^{\prime \prime}\right),\left(b, 2^{\prime \prime}\right)\right\}, H^{0}=\{i "\}_{1}^{5} \cup C_{4}^{0}$ при перетворенні за- 
даному формулою: $\varphi\left(K_{5}+H, \sum_{i=1}^{5}\left(i^{\prime}+i^{\prime \prime}\right)\right) \rightarrow\left(D_{39},\{i\}_{i=1}^{5}\right)$ та виконаному шляхом ототожнення усіх пар $\left(i^{\prime}, i^{\prime \prime}\right)$ множин $M^{\prime}=\left\{i^{\prime}\right\}_{1}^{5}$ та $M^{\prime \prime}=\{i "\}_{1}^{5}$.

\section{Лема 3.}

1) $D_{40}$ - обструкція для тору $€ \varphi$-образом графів $K_{3,3}$ та $H$, де $K_{3,3}^{0}=\left\{i^{\prime}\right\}_{1}^{6}, H^{1}=3 K_{4}^{1}, H^{0}=\left\{i^{\prime \prime}\right\}_{1}^{6} \cup\{a, b, c\}$, причому кожна пара графів $\mathrm{K}_{4}$ матиме тільки одну спільну вершину 3 множини $\{a, b, c\}$, де $H\{a, b, c\}=K_{3}$, при перетворенні заданому наступною формулою: $\varphi\left(K_{3,3}+H, \sum_{i=1}^{6}\left(i^{\prime}+i^{\prime \prime}\right)\right) \rightarrow\left(D_{40},\{i\}_{i=1}^{6}\right)$ шляхом ототожнення усіх пар $\left(i^{\prime}, i^{\prime \prime}\right)$ вершин з множин приєднання $M^{\prime}=\left\{i^{\prime}\right\}_{1}^{6}, M^{\prime \prime}=\left\{i^{\prime \prime}\right\}_{1}^{6}$;

2) $D_{41} \in \quad \varphi$-образ графів $K_{5} \quad$ та $H$, де $H^{0}=\left\{i^{\prime \prime}\right\}_{1}^{5} \cup\{a, b, c, v\}$, $K_{5}^{0}=\left\{i^{\prime}\right\}_{1}^{5}, \quad H^{1}=K_{5}^{1} \backslash\left(v, 2^{\prime \prime}\right) \cup\left\{\left(a, 5^{\prime \prime}\right),\left(c, 5^{\prime \prime}\right),\left(a, 4^{\prime \prime}\right),\left(c, 4^{\prime \prime}\right),\left(b, 3^{\prime \prime}\right),\left(c, 3^{\prime \prime}\right)\right.$, $\left.\left(a, 1^{\prime \prime}\right),\left(b, 1^{\prime \prime}\right)\right\}, H$ - квазізірка 3 центром $K_{1,3}$, який на множині вершин $\left\{i^{\prime \prime}\right\}_{1}^{4} \cup\{a, b, c, v\}$ породжує підграф гомеоморфний $K_{5} \backslash\left(v, 2^{\prime \prime}\right)$, при перетворенні заданому наступною формулою: $\varphi\left(K_{5}+H, \sum_{i=1}^{5}\left(i^{\prime}+i^{\prime \prime}\right)\right) \rightarrow\left(D_{41},\{i\}_{i=1}^{5}\right)$ шляхом ототожнення усіх пар $\left(i^{\prime}, i^{\prime \prime}\right)$ вершин 3 множин приєднання $M^{\prime}=\left\{i^{\prime}\right\}_{1}^{5}, M^{\prime \prime}=\left\{i^{\prime \prime}\right\}_{1}^{5}$;

3) $D_{42}$ - граф-обструкція для тору $\epsilon$-образом графів $K_{5}$, $K_{5}^{0}=\left\{i^{\prime}\right\}_{1}^{5}$, та $H$, де $H-$ квазізірка 3 центром $C_{4}-$ простим циклом довжини 4, який на вершинах $a, b, c, v, 2 "$ породжує підграф гомеоморфний $K_{4}$, де $C_{4}^{1}=\{(a, b),(c, b),(a, v),(c, v)\}, H^{0}=$ $=\left\{i^{\prime \prime}\right\}_{1}^{5} \cup C_{4}^{0}, \quad H^{1}=K_{4}^{1} \cup\left\{(1 ", a),(1 ", b),(3 ", v),(3 ", b),(v, 4 "),(a, 4 "),\left(c, 5^{\prime \prime}\right)\right\}$, при перетворенні заданому формулою: $\varphi\left(K_{5}+H, \sum_{i=1}^{5}\left(i^{\prime}+i^{\prime \prime}\right)\right) \rightarrow$ $\rightarrow\left(D_{42},\{i\}_{i=1}^{5}\right)$ та виконаному шляхом ототожнення усіх пар $\left(i^{\prime}, i^{\prime \prime}\right) 3$ множин $M^{\prime}=\left\{i^{\prime}\right\}_{1}^{5}$ та $M^{\prime \prime}=\left\{i^{\prime \prime}\right\}_{1}^{5}$;

4) $D_{43}$ - обструкція для тору є $\varphi$-образом графів $K_{5}$ та $H$, де $H^{0}=$ $=\left\{i^{\prime \prime}\right\}_{1}^{5} \cup\{a, b, c, v\}, \quad H^{1}=K_{5}^{1} \backslash\left(1 ", 2^{\prime \prime}\right) \cup\left\{(a, 4 "),(c, 4 "),\left(b, 3^{\prime \prime}\right),\left(v, 5^{\prime \prime}\right)\right\}$, 
$K_{5}^{0}=\left\{i^{\prime}\right\}_{1}^{5}, H-$ квазізірка 3 центром $C_{4}$, який на множині вершин $\{i "\}_{1}^{2} \cup\{a, b, c, v\}$ породжує підграф гомеоморфний $K_{5} \backslash(1 ", 2 "), \quad$ при перетворенні заданому формулою: $\varphi\left(K_{5}+H, \sum_{i=1}^{5}\left(i^{\prime}+i^{\prime \prime}\right)\right) \rightarrow\left(D_{43},\{i\}_{i=1}^{5}\right)$ шляхом ототожнення усіх пар $\left(i^{\prime}, i^{\prime \prime}\right)$ вершин з множин приєднання $M^{\prime}=\left\{i^{\prime}\right\}_{1}^{5}, M^{\prime \prime}=\left\{i^{\prime \prime}\right\}_{1}^{5}$.

Лема 4. Виконуються наступні твердження:

1) $D_{44}$ - обструкція для тору є $\varphi$-образом графів $K_{3,3}$ та $H$, де $H^{0}=\{i "\}_{1}^{6} \cup\{a, b, c\}, \quad H^{1}=K_{4}^{1} \backslash(a, c) \cup\left\{\left(a, 1^{\prime \prime}\right),\left(c, 1^{\prime \prime}\right),\left(a, 5^{\prime \prime}\right),\left(b, 5^{\prime \prime}\right)\right.$, $\left.\left(a, 3^{\prime \prime}\right),\left(b, 3^{\prime \prime}\right),\left(c, 2^{\prime \prime}\right),(b, 2 "), \quad\left(c, 4^{\prime \prime}\right),\left(b, 4^{\prime \prime}\right)\right\}, K_{3,3}^{0}=\left\{i^{\prime}\right\}_{1}^{6}, \quad$ де $H\left\{a, b, c, 6 ", 1^{\prime \prime}\right\} \cong K_{4}$, причому вершина $1 "$ розділяє ребро $(a, c)$, при перетворенні заданому наступною формулою: $\varphi\left(K_{3,3}+H, \sum_{i=1}^{6}\left(i^{\prime}+i^{\prime \prime}\right)\right) \rightarrow\left(D_{44},\{i\}_{i=1}^{6}\right)$ шляхом ототожнення усіх пар $\left(i^{\prime}, i^{\prime \prime}\right)$ вершин з множин приєднання $M^{\prime}=\left\{i^{\prime}\right\}_{1}^{6}, M^{\prime \prime}=\left\{i^{\prime \prime}\right\}_{1}^{6}$;

2) $D_{45}$ - обструкція для тору є $\varphi$-образом графів $K_{6} \backslash K_{3}^{1}$ та $H$, де $H^{0}=\{i "\}_{1}^{5} \cup\{a, v, c\} \quad H=S t_{5}(a)+S t_{5}(c)+S t_{3}(v), \quad H^{1}=S t_{5}^{1}(a) \cup$ $\cup S t_{5}^{1}(c) \cup S t_{3}^{1}(v), K_{6}^{0}=\left\{i^{\prime}\right\}_{1}^{6}$, при перетворенні заданому наступною формулою: $\varphi\left(K_{6} \backslash K_{3}^{1}+H, \sum_{i=1}^{6}\left(i^{\prime}+i^{\prime \prime}\right)\right) \rightarrow\left(D_{45},\{i\}_{i=1}^{6}\right)$ шляхом ототожнення усіх пар $\left(i^{\prime}, i^{\prime \prime}\right)$ вершин 3 множин приєднання $M^{\prime}=\left\{i^{\prime}\right\}_{1}^{6}, M^{\prime \prime}=\left\{i^{\prime \prime}\right\}_{1}^{6}$;

3) $D_{46}$ - обструкція для тору $є \varphi$-образом графів $K_{5}$ та $H$, де $H^{0}=\left\{i^{\prime \prime}\right\}_{1}^{5} \cup\left\{a, b, c^{\prime \prime}, v\right\}, K_{5}^{0}=\left\{i^{\prime}\right\}_{1}^{5} \cup\left\{c^{\prime}\right\}$, вершина $c^{\prime}$ розділяє ребpo $\left(1^{\prime}, 3^{\prime}\right), H^{1}=K_{5}^{1} \backslash\left\{\left(1^{\prime \prime}, 3^{\prime \prime}\right)\right\} \cup K_{4}^{1} \cup\left\{\left(a, 4^{\prime \prime}\right),\left(a, 5^{\prime \prime}\right)\right\}, H\left\{2^{\prime \prime}, 3^{\prime \prime}, c^{\prime \prime}, v\right\}=$ $=K_{4} \quad H\left\{1 ", 3 ", v, a, b, c^{\prime \prime}\right\}=K_{5} \backslash(1 ", 3 "), \quad H\left(\left\{4 ", 5^{\prime \prime}, a, b, c "\right\}\right)=K_{2,3}$, $H$ - квазізірка з центром на множині вершин $\{a, b, v\}$ породжує підграф гомеоморфний $K_{3}$, при перетворенні заданому формулою: $\varphi\left(K_{5}+H, \sum_{i=1}^{5}\left(i^{\prime}+i^{\prime \prime}\right),\left(c^{\prime}+c^{\prime \prime}\right)\right) \rightarrow\left(D_{47},\{i\}_{i=1}^{5}, c\right)$ шляхом отото- 
жнення усіх пар $\left(i^{\prime}, i^{\prime \prime}\right)$ вершин з множин приєднання $M^{\prime}=\left\{i^{\prime}\right\}_{1}^{5}$, $M "=\{i "\}_{1}^{5}$, та пари $\left(c^{\prime} c^{\prime \prime}\right)$;

4) $D_{47}$ - обструкція для тору $є \varphi$-образом графів $K_{5}$ та $H$, де $H^{0}=\left\{i^{\prime \prime}\right\}_{1}^{5} \cup\{a, b, c, v\}, K_{5}^{0}=\left\{i^{\prime}\right\}_{1}^{5}, H^{1}=K_{3,3}^{1} \cup K_{2,3}^{1}, K_{3,3}^{0}=\left\{1^{\prime \prime}, 3^{\prime \prime}, v,\right\} \cup$ $\cup\{a, b, c\}, H\left\{1 ", 3^{\prime \prime}, v,\right\}=H\{a, b, c\}=\overrightarrow{K_{3}}, H(\{4 ", 5 ", a, b, c\})=K_{2,3}-$ квазізірка з центром на множині вершин $\{a, b, c, v\}$ породжує підграф гомеоморфний $K_{1,3}$, при перетворенні заданому наступною формулою: $\varphi\left(K_{5}+H, \sum_{i=1}^{5}\left(i^{\prime}+i^{\prime \prime}\right)\right) \rightarrow\left(D_{47},\{i\}_{i=1}^{5}\right)$ шляхом ототожнення усіх пар $\left(i^{\prime}, i^{\prime \prime}\right)$ вершин 3 множин приєднання $M^{\prime}=\left\{i^{\prime}\right\}_{1}^{5}$, $M "=\{i "\}_{1}^{5}$.

Лема 5. Виконуються наступні твердження:

1) $D_{48} \quad \epsilon \quad \varphi$-образом $\quad K_{6} \backslash K_{1,2}^{1} \quad$ та $\quad H$, де $\quad K_{3,3}^{0} \backslash K_{1,2}^{1}=\left\{i^{\prime}\right\}_{1}^{6}$, $H^{0}=\left\{i^{\prime \prime}\right\}_{1, i \neq 2}^{6} \cup\left\{a, b, b^{\prime \prime}\right\}$, вершина $b^{\prime}$ розділяе $\left(2^{\prime}, 6^{\prime}\right), H^{1}=K_{5}^{1} \backslash$ $\backslash\left(5^{\prime \prime}, 6^{\prime \prime}\right) \cup\left\{\left(a, 1^{\prime \prime}\right),\left(b, 1^{\prime \prime}\right),\left(a, 5^{\prime \prime}\right),\left(a, 4^{\prime \prime}\right),\left(c^{\prime \prime}, 3^{\prime \prime}\right)\right\}$, де $H\left\{a, b, c^{\prime \prime}, 6^{\prime \prime}, 5^{\prime \prime}\right\} \cong$ $\cong K_{5} \backslash\left(5^{\prime \prime}, 6^{\prime \prime}\right), \quad$ при перетворенні заданому формулою: $\left.\varphi\left(\left(K_{6} \backslash K_{1,2}^{1}\right)+H,\left(\sum_{i=1, i \neq 2}^{6}\left(i^{\prime}+i^{\prime \prime}\right),\left(b^{\prime}+b^{\prime \prime}\right)\right)\right) \rightarrow\left(D_{48},\left(\{i\}_{i=1, i \neq 2}^{6}, b\right)\right)\right) \quad$ та виконаному шляхом ототожнення усіх пар $\left(i^{\prime}, i^{\prime \prime}\right)$ вершин 3 множин $M^{\prime}=\left\{i^{\prime}\right\}_{1}^{6}, M^{\prime \prime}=\left\{i^{\prime \prime}\right\}_{1}^{6}$ та $\left(b^{\prime}, b^{\prime \prime}\right)$ в $b$, причому вершина 2' стане вершиною 2;

2) $D_{49} \in \varphi$-образом графів $K_{6} \backslash 2 K_{2}^{1}$ та $H$, де $K_{6} \backslash 2 K_{2}^{1}=\left\{i^{\prime}\right\}_{1}^{6}$, $K_{6}^{1} \backslash 2 K_{2}^{1}=K_{6}^{1} \backslash\left\{\left(4^{\prime}, 6^{\prime}\right),\left(2^{\prime}, 5^{\prime}\right)\right\}, H^{0}=\left\{i^{\prime \prime}\right\}_{1}^{6} \cup\{a, b, c\}, H\left(\left\{i^{\prime \prime}\right\}_{1}^{2} \cup\{a, b\}\right)=$ $=K_{4}, H(\{1 ", 2 ", 4 ", 6 " c\})=S t_{4}(c), H^{1}=K_{4}^{1} \cup S t_{4}^{1}(c) \cup\{(a, 6 "),(b, 3 ")$, $\left.(a, 4 "),\left(a, 5^{\prime \prime}\right)\right\}$, при перетворенні заданому формулою: $\left.\varphi\left(\left(K_{6} \backslash 2 K_{2}^{1}\right)+H, \sum_{i=1}^{6}\left(i^{\prime}+i^{\prime \prime}\right)\right) \rightarrow\left(D_{49},\{i\}_{i=1}^{6}\right)\right)$ та виконаному шляхом ототожнення усіх пар $\left(i^{\prime}, i^{\prime \prime}\right)$ вершин 3 множин $M^{\prime}=\left\{i^{\prime}\right\}_{1}^{6}$, $M "=\{i "\}_{1}^{6}$; 
3) $D_{50}$ - $\varphi$-образ графів $K_{6}$ та $H$, де $K_{6}^{0}=\left\{i^{\prime}\right\}_{1}^{6}, H^{0}=\left\{i^{\prime \prime}\right\}_{1}^{6} \cup\{a, b, c\}$, $H\left(\{i "\}_{4}^{5} \cup\{c, b\}\right)=K_{4}, H\left(\left\{1 ", 6 ", 4^{\prime \prime}, 5 " a\right\}\right)=S t_{4}(a), H^{1}=K_{4}^{1} \cup S t_{4}^{1}(a) \cup$ $\cup\left\{\left(c, 2^{\prime \prime}\right),\left(b, 3^{\prime \prime}\right)\right\}$, при перетворенні заданому формулою: $\left.\varphi\left(K_{6}+H, \sum_{i=1}^{6}\left(i^{\prime}+i^{\prime \prime}\right)\right) \rightarrow\left(D_{50},\{i\}_{i=1}^{6}\right)\right)$ та виконаному шляхом ототожнення усіх пар $\left(i^{\prime}, i^{\prime \prime}\right)$ вершин з множин $M^{\prime}=\left\{i^{\prime}\right\}_{1}^{6}, M^{\prime \prime}=\left\{i^{\prime \prime}\right\}_{1}^{6}$;

4) $D_{51}$ - обструкція для тору $є \varphi$-образом графів $K_{5}$ та $H$, де $H^{0}=\left\{i^{\prime \prime}\right\}_{1}^{5} \cup\{a, b, c, v\}, K_{5}^{0}=\left\{i^{\prime}\right\}_{1}^{5}, H^{1}=H_{1}^{1} \cup H_{2}^{1} \cup\left\{\left(b, 1^{\prime \prime}\right),\left(v, 3^{\prime \prime}\right)\right\}$, $H_{1}^{0}=\left\{4^{\prime \prime}, 5^{\prime \prime}, c, b, v\right\}, H_{2}^{1}=\left\{2^{\prime \prime}, 4^{\prime \prime}, 5^{\prime \prime}, a, c\right\}, H_{1}=H(\{4 ", 5 ", c, b, v\})=$ $=K_{5} \backslash(4 ", 5 "), \quad H_{2}=H(\{2 ", 4 ", 5 ", a, c\})=K_{5} \backslash(4 ", 5 "), \quad$ причому $H_{2}$ має $3 K_{5}$ спільний ланцюг довжини 2 на вершинах $\{4 ", 5 ", c\}$, де $H$ - квазізірка 3 центром на множині вершин $\{a, b, c, v\}$ на якій породжує підграф $K_{4} \backslash K_{1,2}$, при перетворенні заданому наступною формулою: $\varphi\left(K_{5}+H, \sum_{i=1}^{5}\left(i^{\prime}+i^{\prime \prime}\right)\right) \rightarrow\left(D_{51},\{i\}_{i=1}^{5}\right)$ шляхом ототожнення усіх пар $\left(i^{\prime}, i^{\prime \prime}\right)$ вершин 3 множин приєднання $M^{\prime}=\left\{i^{\prime}\right\}_{1}^{5}, M^{\prime \prime}=\{i "\}_{1}^{5}$;

Із вищенаведених лем 1-5 випливатиме основний результат.

Теорема. Кожна граф-обструкція роду $2 D_{32}, \ldots, D_{51}$ [5] на 9-ти вершинах $є$ результатом $\varphi$-перетворення трьох зв'язних графів $X, Y, Z$, які задовольняють одному з наступних випадків:

1) граф $Y$ гомеоморфний $K_{5}$ чи $K_{3,3}$ (можливо із кількома додатковими ребрами) вкладений в тор $\sigma$, граф $Z$ відсутній, а інший граф $X$ є або площинним 2-мінімальним відносно множини точок приєднання до графа $Y$ на недвоклітці $\sigma \backslash Y$ із нульовими характеристиками $\theta$ та $\partial \theta$ для множини точок приєднання до графа $Y$, або площинним 3-мінімальним на $s$ недвоклітці тора, $s \in \sigma_{1} \backslash Y$, із характеристиками $\theta, \partial \theta$, де $\theta=1$ чи $\partial \theta=1$, для множини точок приєднання графа $X$ до графа $Y$;

2) граф $Y$ один з графів $K_{5}$ чи $K_{3,3}$, можливо без ребра, вкладений в тор $\sigma$, а інший граф $X$ роду $1 € 2$-мінімальним відносно множини точок приєднання на недвоклітці $\sigma \backslash Y$ із нульовими характеристиками $\theta, \partial \theta$ множини точок приєднання графа $X$ до графа $Y$, граф $Z$ відсутній; 
3) граф $Y$ містить частину гомеоморфну $K_{5}$ чи $K_{3,3}$ (можливо із кількома додатковими ребрами) вкладений в тор $\sigma$, граф $Z-$ проста зірка, граф $X \in$ площинною квазізіркою із центральним графом $M$ на двох вершинах, яка не $\epsilon$ 2-мінімальним графом на недвоклітці $s, s \in \sigma \backslash Y$, причому існує, принаймні одна, пара вершин простої зірки $Z$, сформована із елементів множини приєднання графа $X$ до графа $Y$, що розділяє на $\partial s$ пару кінцевих вершин 3 множини приєднання графа $X$ до графа $Y$.

\section{Список використаних джерел:}

1. Хоменко М. П. $\varphi$-перетворення графів. Київ, 1971.378 с.

2. Петренюк В. І. Построение графов-обструкций ограниченного ориентируемого рода. XVI Международная конферениия «Проблемы теоретической кибернетики». Нижний Новгород, 2011. С. 363-368.

3. Hur Suhjin. The Kuratowski covering conjecture for graphs of order less than 10. PhD dissertation, Ohio State University, 2008.

4. Петренюк В. І. Структура 28-ми 9-ти вершинних графів-обструкцій тора. Математичне та комп'ютерне моделювання. Серія фізико-математичних наук. Кам'янець-Подільський національний університет імені Івана Огієнка. 2017. Т. 16. С. 145-151.

\section{STRUCTURE OF 20 9-VERTECES GRAPHS OBSTRUCTIONS FOR TORUS}

Structure all 9-verteces graphs obstructions for torus was found.

Key words: structure, 9-verteces graph obstructions, torus, $\varphi$-transformation.

Одержано 14.01.2019 